\title{
Doctors' versus patients' evaluation of results after
} neurosurgery

\author{
HANS CARLSON, LUIGI PELLETTIERI \\ From the Department of Neurosurgery, University Hospital, Uppsala, Sweden
}

SUMMARY A comparison between doctors' and patients' judgements of results after treatment for various neurosurgical conditions has been made. Ninety consecutive patients (41 women and 49 men) treated for trauma, vascular diseases, tumours, pain, and malformations were included in the study. The physicians responsible for the treatment and the patients rated the result of the therapeutic efforts (operative or nonoperative) independently from each other on a five grade scale at the time for discharge. A follow-up study was also made 8-24 months after the treatment. Three different groups of paired observations were distinguished: (1) "identical opinion", (2) "pessimistic opinion" (physician's score lower than patient's) and (3) "optimistic opinion" (physician's score higher than patient's). At the time of discharge identical opinion about the result of treatment was present in $83 \%$ ( 75 cases) whereas $9 \%$ ( 8 cases) and $8 \%$ ( 7 cases) were referred to the groups of pessimistic and optimistic opinions respectively. In the follow up series the corresponding values were $57 \%, 12 \%$, and $31 \%$. The increase of non-identical judgements in the follow-up study was found almost exclusively in the group of patients treated for painful conditions. Also the spread of judgements was largest in the group of painful conditions. Otherwise there was no correlation between the actual result of treatment and diagnosis, sex or age.

The medical decision making procedure is often based on analogous assumptions. This technique offers a certain amount of security in predicting the consequences of therapeutic efforts. Thus, medical decisions like others are made upon an analysis of multiple factors on the one hand and known results/consequences on the other. Certain factors are ascribed a high prognostic value whereas others contribute to a smaller degree. The prognostic value of a specific factor should be influenced by the experiences of the individual physician and by known relations depicted from the medical literature. Likewise in judging the results of a therapy the doctors can evaluate them on the basis of own experience and scientifical knowledge. The patient on the other hand weighs the reality of results against wanted/expected outcome. Therefore in assessing clinical results it can be expected that the opinion of the physician and of the patient may diverge in situations involving judgements of residual symptoms and signs as well as of the therapeutic results. (See, for example, ref. 1).

Address for reprint requests: Dr Luigi Pellettieri, Department of Neurosurgery, University Hospital, S-751 85 Uppsala, Sweden.

Received 18 September 1987 and in final revised form 15 August 1988.
The purpose of this investigation was to elucidate whether the evaluation of results after treatment, for a variety of neurosurgical conditions differed between the physician responsible for the treatment and the patient.

\section{Patients and methods}

Ninety patients and 10 physicians responsible for the treatment at the Neurosurgical Department, University Hospital, Uppsala, took part in the investigation. The patients, 41 women and 49 men, were distributed among the following diagnostic groups; Trauma: 12, Vascular diseases: 14. Tumours of the brain and the spinal cord: 29, Painful conditions and degenerative diseases in the locomotor apparatus: 25, Malformations and cases for reconstructive surgery: 10.

Seventy-one patients were subjected to surgical procedures and 19 were treated according to conservative procedures (non-operative therapies; physiotherapy, transcutaneous nerve stimulation etc). For practical reasons the investigation was made during two short periods on consecutive cases. The patient and the physician responsible for the treatment were supplied with a questionnaire with the following alternatives for judging the result of the treatment; very good, good, fair, bad, very bad, corresponding to a numerical scale from 5-1. The patient and the physician were instructed to make their 
complete the questionnaire on the day of discharge. It was made clear to the patient and to the doctor that their judgements should be made independently and not after discussion. Moreover, the patients were told not to include an opinion about general care in the ward. Disoriented patients were excluded from the investigation. An identical follow-up questionnaire was sent to the patients 8-24 months after discharge. A total of 69 patients took part (53 surgically treated and 16 conservative) in this follow-up study. Thus, 21 patients were missing. Of these, 6 could not be contacted and 2 had died from other disorders. Finally, 13 patients with malignant gliomas were excluded from this follow-up study since the natural course of this disease is known to be extremely poor. The exclusion of these patients does not have any bearing on the results or conclusions of the study (as a whole).

The degree of similarity between judgements made by patients and doctors has been estimated. Three different groups of paired observations have been distinguished.

(1) "Identical opinion". This group includes pairs in which a complete match between the two judgements occurred and pairs in which the doctor had scored the result as good and the patient had scored it as very good $(4,5)$.

(2) "Pessimistic opinion". This group includes pairs in which the score of the doctor was one grade or more below the score given by the patient (except for pairs $4,5 \mathrm{cf}$ above).

(3) "Optimistic opinion" This group includes pairs where the score given by the doctor exceeded the patient score by one grade or more.

The material has been analysed with respect to possible differences between the various diagnostic groups. Conventional statistical methods ( $\chi^{2}$-test, logistic regression) have been used for the evaluation.

\section{Results}

The table shows the distribution of the three different categories of treatment evaluation (cf. methods). Of the 90 paired observations made at the time of discharge 75 cases $(83 \%)$ were "identical". The "pessimistic opinion" and "optimistic opinion" groups amounted to eight cases $(9 \%)$ and seven cases $(8 \%)$, respectively. The corresponding values for the followup series were 40 identical pairs $(57 \%)$, eight "pessimistic" judgements (12\%) and 21 "optimistic" judgements $(31 \%)$. Thus, the degree of conformity in the judgement of the results between the physician and the patient decreased considerably at the time for follow up $(p<0.001)$. The number of "optimistic" evaluations increased significantly $(p<0.001)$. The decrease in conformity was found to be largely dependent on corresponding increase in "optimistic" judgements in the diagnostic group "painful and degenerative conditions" (from 3 to 10 cases). In the other diagnostic groups such changes were less marked. However, in the tumour group a large number dropped out (13 cases, cf Methods). Therefore an increase of "optimistic" opinions from two to five cases in this group should be regarded as considerable. Judgements of the result of treatment were not
Table Distribution into three different groups of evaluation at discharge and at follow up (see text)

\begin{tabular}{llll}
\hline & Identical & $\begin{array}{l}\text { Doctor } \\
\text { optimistic }\end{array}$ & $\begin{array}{l}\text { Doctor } \\
\text { pessimistic }\end{array}$ \\
\hline At Discharge $(\mathrm{n}=90)$ & 75 & 7 & 8 \\
At Follow-up $(\mathrm{n}=69)$ & 40 & 21 & 8 \\
\hline
\end{tabular}

dependent on the sex or age of the patients (see ref 2). Furthermore, no particular evaluation correlating to diagnosis has been established. However, the spread of judgements was largest within the group of painful and degenerative conditions. In the present study the results were estimated as good or very good in nearly $80 \%$ of the total cases. This value mainly reflects the selection criteria of the patients and should not be interpreted as evidence for extremely good results of treatment procedures in themselves for a variety of neurosurgical conditions.

\section{Discussion}

The present investigation reveals a high degree of conformity in the treatment evaluation made indepen -0 dently by the patient and the physician. About $80 \%$ o류 the judgements can be regarded as identical. This 을 figure indicates that the patients were given adequate information about the condition and the therapeutie $T$ goals.

The first evaluation (made at the time of discharge may be regarded as premature and non-representative of a true result. Factors unrelated to the actual resulf could have influenced the judgement at this point. Important factors are probably the psychological environment of the patient and the comparatively limited demands of the patients staying in a ward. ${ }^{2}$ These factors, together with optimistic judgements made by the physicians, could lead to a false opinion of the therapeutic result. Disagreement in the evaluation made by the physician and the patient at the time of discharge could be due to several factors. The most relevant situation is when the patient has been given inappropriate information about the condition and the therapeutic possibilities. Another factor, which could give rise to a different judgement is an overestimation of what modern medicine and recent technical procedures/equipment can achieve. This factor is, of course, a source of poorly founded optimism among patients as well as among physicians.

In the follow-up survey (8-24 months after discharge) similarity of the judgements was less marked. An increased divergence of opinions was found in the group of painful and degenerative conditions. In the other diagnostic groups no such divergence was observed. This relation merely reflects the nature of these disabling conditions with recurring symptoms. 
The present study has shown that a treatment given according to clinical experience may produce identical judgements of the results by the patient and by the physician. A pre-requisite for identical opinions is adequate information about the therapy and its possible consequences. ${ }^{3-5}$ This information should, preferentially, be given by the doctor directly to the patient. When informing patients suffering from painful conditions or degenerative conditions of the locomotor apparatus the risk of recurring symptoms/ signs should be considered.

Patients in coma or presenting disorientation at income were excluded from the study. Those recovering from these conditions after adequate therapy may judge the result of the treatment from a completely different point of view than the physician.

\section{References}

1 Speedling EJ, Rose DN. Building an effective doctorpatient relationship: From patient satisfaction to patient participation. Soc Sci Med 1985;21:115-20.

2 Carmel S. Satisfaction with hospitalization: A comparative analysis of three types of services. Soc Sci Med 1985;21:1243-49.

3 McGhee A. The Patient's Attitude to Nursing Care. 1961; London: Livingstone.

4 Cartwright A. Human Relations and Hospital Care. 1964; London: Routledge and Kegan Paul.

5 Houston C, Pasanen W. Patients perception of hospital care. Hospitals 1974;46:70-74.

\section{Sir Victor Horsley's surgery for epilepsy}

The first operation for a brain tumour' was probably performed by William Macewen on 27 July 1879 . The patient was a 14 year old girl with a left frontal convexity meningioma. By 1888, Macewen had reported another 11 brain operations, mostly for cysts, abscesses, tuberculomata, depressed fractures and haematoma. The most celebrated, but not the first operation, was the removal of a frontal glioma from the right ascending parietal convolution, on 25 November 1884 by Rickman Godlee, the nephew of Lord Lister.

Sir Victor Horsley (1857-1916) was a remarkable, versatile and individualistic genius. He is renowned for many feats of original surgery and for localisation of cortical physiology in collaboration, inter alia, with such giants as David Ferrier, Jackson, Beevor, Gowers, Clarke and Kinnier Wilson. His first surgery on the brain can be seen as the forerunner of modern surgery for epilepsy. His first patient James B, aged 22 years had sustained an infected depressed fracture at the age of 7 and later developed prolonged attacks of Jacksonian status epilepticus. On 25 May 1886 Horsley exposed the obvious lesion and removed a highly vascular scar 3 by $2 \mathrm{~cm}$. The fits did not recur. Hughlings Jackson and David Ferrier were in the theatre, as they had been for Godlee's operation. Jackson then suggested surgery for another of his patients, a Thomas W. in whom he had diagnosed epilepsy due to a cortical tuberculoma. Jackson proved correct, the lesion was excised and the patient survived another eight years. Horsley's third case was a stable boy who had endured a number of injuries to the head and consequent epilepsy. He had a tender old depressed fracture of the left parietal convexity and Horsley associated this with his unusual "rectal aura". Extensive debridement of the cortex and inner table of the skull was a success.

Horsley's incredible energies and achievements are delightfully described by Irving Cooper ${ }^{2}$ and by Stephen Paget ${ }^{3}$ and JB Lyons. ${ }^{4} \mathrm{He}$ was named Victor Alexander after Queen Victoria at her own suggestion and passed on by a lady in waiting who was friend to Horsley's mother. He died tragically, aged 59, of fever, probably heat stroke in Amara on the Tigris campaign, for which he had courageously but unwisely volunteered at the age of 57 , in 1914 .

\section{References}

1 Pearce JMS. The first attempts at removal of brain tumours. In: FC Rose and WF Bynum, eds. Historical Aspects of the Neurosciences, A Fetschrift for Macdonald Critchley. New York: Raven Press, 1982:239-43.

2 Cooper IS. Sir Victor Horsley: Father of Modern Neurological Surgery. ibid 235-39.

3 Paget S. Sir Victor Horsley. London: Constable \& Co, 1919.

4 Lyons JB. The Citizen Surgeon: A biography of Sir Victor Horsley. London: Dawnay, 1966. 